\title{
Towards the Unification, Part 2: Simplified Equations, Covariant Derivative, Photons
}

\author{
Claude Daviau${ }^{1}$, Jacques Bertrand ${ }^{2}$, Dominique Girardot ${ }^{3}$ \\ ${ }^{1}$ Le Moulin de la Lande, 44522, Pouillé-les-Coteaux, France \\ ${ }^{2} 15$ Avenue Danielle Casanova, 95210, Saint-Gratien, France \\ ${ }^{3} 95$ Rue Marceau, 91120, Palaiseau, France \\ Email:claude.daviau@nordnet.fr, bertrandjacques-m@orange.fr,dominique.girardot2@sfr.fr
}

How to cite this paper: Daviau, C., Bertrand, J. and Girardot, D. (2016) Towards the Unification, Part 2: Simplified Equations, Covariant Derivative, Photons. Journal of Modern Physics, 7, 2398-2417.

http://dx.doi.org/10.4236/jmp.2016.716207

Received: November 22, 2016

Accepted: December 27, 2016

Published: December 30, 2016

Copyright $\odot 2016$ by authors and Scientific Research Publishing Inc. This work is licensed under the Creative Commons Attribution International License (CC BY 4.0).

http://creativecommons.org/licenses/by/4.0/

\begin{abstract}
We continue the study of the Standard Model of Quantum Physics in the Clifford algebra of space. We get simplified mass terms for the fermion part of the wave. We insert the simplified equations in the frame of General Relativity. We construct the electromagnetic field of the photon, alone boson without proper mass. We explain how the Pauli principle comes from the equivalence principle of General Relativity. We transpose in the frame of the algebra of space the second quantification of the electromagnetic field. We discuss the changes introduced here.
\end{abstract}

\section{Keywords}

Boson, Clifford Algebra, Dirac Equation, Electromagnetism, Electron, Gravitation, Magnetic Monopole, Neutrino, Photon, Quark, Relativistic İnvariance, Strong İnteractions, Weak İnteractions

\section{Reduced Equations}

We use the introduction, the notations and results of the first part [1]. We have explained there how the Lagrangian density is both consequence and cause of the equations ruling the waves of the electron, its neutrino and the $d$ and $u$ quarks with their three states of color. These particles are those of the first generation in the Standard Model of Quantum Physics (SM). The self-causality of this part of the SM, main result of [1], explains why a principle of extremum exists in physics. This self-causality is also the limit of the domain where the Lagrangian mechanism is acting: contrary to the SM where the Lagrangian density has a fermion and a boson part, we cannot get the selfcausality of a boson part of the Lagrangian density. Then we must get all properties of 
the boson part of the SM only from the wave equations of the fermion part.

The $1+3$ boson fields of the $U(1) \times S U(2)$ gauge group of electro-weak interactions and the 8 bosons named "gluons" of the $S U$ (3) group of chromodynamics generate the $U(1) \times S U(2) \times S U(3)$ gauge group of the SM. They must account for the invariance under $C l_{3}^{*}=G L(2, \mathbb{C})$. This group generalizes the $S L(2, \mathbb{C})$ group which is used to get the relativistic invariance of the Dirac wave equation [2]. We recall that the dilation reads $D: x \mapsto x^{\prime}=M x \tilde{M}$ where $M$ is any element in $C l_{3}^{*}$ and $\tilde{M}=M^{\dagger}$ [3]-[16]. The vectors transforming like $x$ which satisfy:

$$
x^{\prime}=M x \tilde{M}=x^{\prime \mu} \sigma_{\mu}=D_{v}^{\mu} x^{v} \sigma_{\mu} ; x^{\prime \mu}=D_{v}^{\mu} x^{v}
$$

are named contravariant. The vectors transforming like the gradient $\nabla=\sigma^{\mu} \partial_{\mu}$ are named covariant. An example of covariant vector is $q A$, the potential space-time vector of the Dirac equation which satisfies:

$$
q A=\bar{M} q^{\prime} A^{\prime} \hat{M} ; q A_{\mu}=D_{\mu}^{v} q^{\prime} A_{v}^{\prime} ; \quad \bar{M}=\widetilde{\widehat{M}} .
$$

Each Dirac wave is made of a right and a left wave. For instance the $\phi^{1}$ wave of the electron reads, with the usual notation of the complex conjugate:

$$
\begin{aligned}
& \phi^{1}=\sqrt{2}\left(\begin{array}{cc}
\xi_{1}^{1} & -\bar{\eta}_{2}^{1} \\
\xi_{2}^{1} & \bar{\eta}_{1}^{1}
\end{array}\right) ; \hat{\phi}^{1}=\sqrt{2}\left(\begin{array}{cc}
\eta_{1}^{1} & -\bar{\xi}_{2}^{1} \\
\eta_{2}^{1} & \bar{\xi}_{1}^{1}
\end{array}\right) \\
& \phi^{1}=R^{1}+L^{1} ; \quad R^{1}=\phi^{1} \frac{1+\sigma_{3}}{2}=\sqrt{2}\left(\begin{array}{cc}
\xi_{1}^{1} & 0 \\
\xi_{2}^{1} & 0
\end{array}\right) ; \quad \hat{L}^{1}=\hat{\phi}^{1} \frac{1+\sigma_{3}}{2}=\left(\begin{array}{cc}
\eta_{1}^{1} & 0 \\
\eta_{2}^{1} & 0
\end{array}\right) .
\end{aligned}
$$

We use similar notations for the wave $\tilde{\phi}^{8}$ of the magnetic monopole, the waves $\phi^{n}, n=2,3,4$ of the $\mathrm{d}$ quark and the waves $\tilde{\phi}^{3+n}, n=2,3,4$ of the u quark (see [1] (2.6)-(2.8)). Right and left waves transform differently under the invariance group:

$$
R^{1} \mapsto R^{\prime 1}=M R^{1} ; \hat{L}^{1} \mapsto \hat{L}^{\prime 1}=\hat{M} \hat{L}^{1} ; R^{8} \mapsto R^{\prime 8}=R^{8} M ; \hat{L}^{8} \mapsto \hat{L}^{\prime 8}=\hat{L}^{8} \hat{M} .
$$

Each chiral wave allows us to construct a unique contravariant vector:

$$
D_{R}^{1}=R^{1} \tilde{R}^{1} ; D_{L}^{1}=L^{1} \tilde{L}^{1} ; D_{R}^{8}=\tilde{R}^{8} R^{8} ; D_{L}^{8}=\tilde{L}^{8} L^{8} .
$$

\subsection{The Lepton Sector}

The wave equations of the electron and of the Lochak's magnetic monopole [17]-[22] use the $a_{j}, j=1, \cdots, 6$ of [1] (3.26) and the potentials of [1] (3.20) and (3.22). They read:

$$
\begin{gathered}
0=\hat{\nabla} R^{1}+2 i \hat{\mathrm{b}} R^{1}+i \frac{m}{\rho}\left(a_{1} \hat{L}^{1}+a_{3} \bar{L}^{8}-a_{5} \bar{R}^{8} \sigma_{1}\right) . \\
0=\bar{\nabla} \tilde{R}^{8}-i\left(\frac{1}{2}+k\right) \hat{\mathrm{b}} \tilde{R}^{8}+i \frac{m}{\rho}\left(a_{6} \bar{L}^{8}+a_{4} \hat{L}^{1}+a_{5} \hat{R}^{1} \sigma_{1}\right) ; k=\frac{\sqrt{3}}{2 \alpha} . \\
0=\nabla \hat{L}^{1}+i\left(\mathrm{~b}+w^{3}\right) \hat{L}^{1}-i\left(w^{1}+i w^{2}\right) \bar{L}^{8}+i \frac{m}{\rho}\left(\bar{a}_{1} R^{1}+\bar{a}_{2} \tilde{L}^{8} \sigma_{1}+\bar{a}_{4} \tilde{R}^{8}\right) . \\
0=\tilde{\nabla} \bar{L}^{8}+i\left(\mathrm{~b}-w^{3}\right) \bar{L}^{8}-i\left(w^{1}-i w^{2}\right) \hat{L}^{1}+i \frac{m}{\rho}\left(\bar{a}_{6} \tilde{R}^{8}-\bar{a}_{2} L^{1} \sigma_{1}+\bar{a}_{3} R^{1}\right) .
\end{gathered}
$$


We do not change anything in (1.6), (1.8) and (1.9) from the wave equations of the $\mathrm{SM}$, where $R^{8}$ is useless and often canceled. We put in (1.7) a term of chiral gauge. The fine structure constant $\alpha$ gives the magnetic charge $g$ of the Dirac magnetic monopole:

$$
Q=\frac{g}{\hbar c} ; Q B=\frac{q}{2 \alpha} B=\frac{\sqrt{3}}{2 \alpha} \mathrm{b} .
$$

We also use the value of the Weinberg-Salam angle obtained in [23] from the properties of the electron. This implies:

$$
\mathrm{b}=\frac{q}{3}\left(2 A-W^{3}\right), \quad g_{2}=2 q=\frac{2 e}{\hbar c}
$$

where $A$ is the electromagnetic potential and $e=q \hbar c$ is the charge of the electron. Two conservative currents exist, the $J_{l}$ and the $K_{l}$ currents generalizing the currents of the Lochak's magnetic monopole and of the Dirac's electron [17]-[22]. They read in the lepton case:

$$
J_{l}=D_{R}^{1}+D_{R}^{8}+D_{L}^{1}+D_{L}^{8} ; \quad K_{l}=D_{R}^{1}+D_{R}^{8}-D_{L}^{1}-D_{L}^{8} .
$$

We explain below why the electromagnetic potential $A$ generalizes the $K_{l}$ current. We first explain how the $J_{l}$ current simplifies the mass terms of our wave equations. We have:

$$
\hat{J}_{l} R^{1}=\left(\hat{R}^{1} \bar{R}^{1}+\bar{R}^{8} \hat{R}^{8}+\hat{L}^{1} \bar{L}^{1}+\bar{L}^{8} \hat{L}^{8}\right) R^{1}=\hat{R}^{1} \bar{R}^{1} R^{1}+\bar{R}^{8} \hat{R}^{8} R^{1}+\hat{L}^{1} \bar{L}^{1} R^{1}+\bar{L}^{8} \hat{L}^{8} R^{1} .
$$

We have also:

$$
\begin{aligned}
& \bar{R}^{1} R^{1}=0 ; \\
& \hat{R}^{8} R^{1}=-a_{5} \frac{\sigma_{1}-i \sigma_{2}}{2} ; \bar{R}^{8} \hat{R}^{8} R^{1}=-a_{5} \bar{R}^{8} \sigma_{1} ; \\
& \bar{L}^{1} R^{1}=a_{1} \frac{1+\sigma_{3}}{2} ; \hat{L}^{1} \bar{L} R^{1}=a_{1} \hat{L}^{1} ; \\
& \hat{L}^{8} R^{1}=a_{3} \frac{1+\sigma_{3}}{2} ; \bar{L}^{8} \hat{L}^{8} R^{1}=a_{3} \bar{L}^{8} .
\end{aligned}
$$

We then get:

$$
\hat{J}_{l} R^{1}=a_{1} \hat{L}^{1}+a_{3} \bar{L}^{8}-a_{5} \bar{R}^{8} \sigma_{1} .
$$

A similar calculation gives:

$$
\begin{aligned}
& \hat{J}_{l} \tilde{R}^{8}=a_{6} \bar{L}^{8}+a_{4} \hat{L}^{1}+a_{5} \hat{R}^{1} \sigma_{1}, \\
& J_{l} \hat{L}^{1}=\bar{a}_{1} R^{1}+\bar{a}_{2} \tilde{L}^{8} \sigma_{1}+\bar{a}_{4} \tilde{R}^{8}, \\
& J_{l} \bar{L}^{8}=\bar{a}_{6} \tilde{R}^{8}-\bar{a}_{2} L^{1} \sigma_{1}+\bar{a}_{3} R^{1} .
\end{aligned}
$$

In each of the four wave Equations (1.6)-(1.9) the mass term has three parts while the $J_{l}$ current is made of four chiral currents. The reason is the cancellation of the terms $\bar{R}^{1} R^{1}, \hat{R}^{8} \tilde{R}^{8}, \bar{L} L^{1}, \hat{L}^{8} \tilde{L}^{8}$. This cancellation also occurs in the calculation of the gauge terms, and the result is: the action of a gauge potential upon any of the chiral $R^{n}$ and $L^{n}$ terms is "exterior" to this part of the wave, caused by the other chiral waves.

We have obtained in [23] Equations. (2.5), (2.8) and (6.10): 


$$
W^{1}=D_{L}^{18}=L^{1} L^{8}+\tilde{L}^{8} \tilde{L}^{1} ; W^{2}=d_{L}^{18}=i L^{1} L^{8}-i \tilde{L}^{8} \tilde{L}^{1} ; W^{3}=-D_{L}^{1}+D_{L}^{8} .
$$

With the value obtained in [23] for the Weinberg-Salam angle we also get $w^{j}=q W^{j}$ and the electro-weak terms present in the wave equations may be simplified:

$-\left(w^{1}+i w^{2}\right) \bar{L}^{8}=2 q\left(-D_{L}^{1}+D_{L}^{8}\right) \hat{L}^{1}=2 w^{3} \hat{L}^{1} ;\left(w^{1}-i w^{2}\right) \hat{L}^{1}=2 q\left(-D_{L}^{1}+D_{L}^{8}\right) \bar{L}^{8}=2 w^{3} \bar{L}^{8}$.

The system (1.6)-(1.9) is then reduced to:

$$
\begin{aligned}
& 0=\left(\hat{\nabla}+2 i \hat{\mathrm{b}}+i \frac{m}{\rho} \hat{J}_{l}\right) R^{1} . \\
& 0=\left(\bar{\nabla}-i\left(\frac{1}{2}+k\right) \hat{\mathrm{b}}+i \frac{m}{\rho} \hat{J}_{l}\right) \tilde{R}^{8} . \\
& 0=\left[\nabla+i\left(\mathrm{~b}+3 w^{3}\right)+i \frac{m}{\rho} J_{l}\right] \hat{L}^{1} . \\
& 0=\left[\tilde{\nabla}+i\left(\mathrm{~b}-3 w^{3}\right)+i \frac{m}{\rho} J_{l}\right] \vec{L}^{8} .
\end{aligned}
$$

We have:

$$
\begin{aligned}
& R^{1}=\sqrt{2}\left(\begin{array}{cc}
\xi_{1}^{1} & 0 \\
\xi_{2}^{1} & 0
\end{array}\right) ; \tilde{R}^{8}=\sqrt{2}\left(\begin{array}{cc}
\xi_{1}^{8} & 0 \\
\xi_{2}^{8} & 0
\end{array}\right) ; \hat{L}^{1}=\sqrt{2}\left(\begin{array}{cc}
\eta_{1}^{1} & 0 \\
\eta_{2}^{1} & 0
\end{array}\right) ; \bar{L}^{8}=\sqrt{2}\left(\begin{array}{cc}
\eta_{1}^{8} & 0 \\
\eta_{2}^{8} & 0
\end{array}\right) ; \\
& D_{R}^{1}=R^{1} \tilde{R}^{1}=2\left(\begin{array}{cc}
\xi_{1}^{1} \bar{\xi}_{1}^{1} & \xi_{1}^{1} \bar{\xi}_{2}^{1} \\
\xi_{2}^{1} \bar{\xi}_{1}^{1} & \xi_{2}^{1} \bar{\xi}_{2}^{1}
\end{array}\right) ; D_{R}^{8}=\tilde{R}^{8} R^{8}=2\left(\begin{array}{cc}
\xi_{1}^{8} \bar{\xi}_{1}^{8} & \xi_{1}^{8} \bar{\xi}_{2}^{8} \\
\xi_{2}^{8} \bar{\xi}_{1}^{8} & \xi_{2}^{8} \bar{\xi}_{2}^{8}
\end{array}\right) ; \\
& D_{L}^{1}=L^{1} \tilde{L}^{1}=2\left(\begin{array}{cc}
\eta_{2}^{1} \bar{\eta}_{2}^{1} & -\eta_{1}^{1} \bar{\eta}_{2}^{1} \\
-\eta_{2}^{1} \bar{\eta}_{1}^{1} & \eta_{1}^{1} \bar{\eta}_{1}^{1}
\end{array}\right) ; D_{L}^{8}=\tilde{L}^{8} L^{8}=2\left(\begin{array}{cc}
\eta_{2}^{8} \bar{\eta}_{2}^{8} & -\eta_{1}^{8} \bar{\eta}_{2}^{8} \\
-\eta_{2}^{8} \bar{\eta}_{1}^{8} & \eta_{1}^{8} \bar{\eta}_{1}^{8}
\end{array}\right)
\end{aligned}
$$

These chiral currents satisfy:

$$
\begin{aligned}
& D_{R}^{10}=\xi_{1}^{1} \bar{\xi}_{1}^{1}+\xi_{2}^{1} \bar{\xi}_{2}^{1} ; \quad D_{R}^{13}=\xi_{1}^{1} \bar{\xi}_{1}^{1}-\xi_{2}^{1} \bar{\xi}_{2}^{1} ; \quad D_{R}^{11}=\xi_{2}^{1} \bar{\xi}_{1}^{1}+\xi_{1}^{1} \bar{\xi}_{2}^{1} ; \quad i D_{R}^{12}=\xi_{2}^{1} \bar{\xi}_{1}^{1}-\xi_{1}^{1} \bar{\xi}_{2}^{1}, \\
& D_{R}^{80}=\xi_{1}^{8} \bar{\xi}_{1}^{8}+\xi_{2}^{8} \bar{\xi}_{2}^{8} ; \quad D_{R}^{83}=\xi_{1}^{8} \bar{\xi}_{1}^{8}-\xi_{2}^{8} \bar{\xi}_{2}^{8} ; \quad D_{R}^{81}=\xi_{2}^{8} \bar{\xi}_{1}^{8}+\xi_{1}^{8} \bar{\xi}_{2}^{8} ; \quad i D_{R}^{82}=\xi_{2}^{8} \bar{\xi}_{1}^{8}-\xi_{1}^{8} \bar{\xi}_{2}^{8}, \\
& D_{L}^{10}=\eta_{1}^{1} \bar{\eta}_{1}^{1}+\eta_{2}^{1} \bar{\eta}_{2}^{1} ; \quad D_{L}^{13}=-\eta_{1}^{1} \bar{\eta}_{1}^{1}+\eta_{2}^{1} \bar{\eta}_{2}^{1} ; \quad D_{L}^{11}=-\eta_{1}^{1} \bar{\eta}_{2}^{1}-\eta_{2}^{1} \bar{\eta}_{1}^{1} ; i D_{L}^{12}=\eta_{1}^{1} \bar{\eta}_{2}^{1}-\eta_{2}^{1} \bar{\eta}_{1}^{1}, \\
& D_{L}^{80}=\eta_{1}^{8} \bar{\eta}_{1}^{8}+\eta_{2}^{8} \bar{\eta}_{2}^{8} ; \quad D_{L}^{83}=-\eta_{1}^{8} \bar{\eta}_{1}^{8}+\eta_{2}^{8} \bar{\eta}_{2}^{8} ; \quad D_{L}^{81}=-\eta_{1}^{8} \bar{\eta}_{2}^{8}-\eta_{2}^{8} \bar{\eta}_{1}^{8} ; i D_{L}^{82}=\eta_{1}^{8} \bar{\eta}_{2}^{8}-\eta_{2}^{8} \bar{\eta}_{1}^{8} .
\end{aligned}
$$

From the previous equalities we can get the result: the chiral currents are isotropic, and using the numeric equations equivalent to (1.19) we get the law of conservation of these four chiral currents. For instance the $D_{R}^{1}$ current satisfies (see [1] (3.40)):

$$
0=\left(D_{R}^{10}\right)^{2}-\left(D_{R}^{11}\right)^{2}-\left(D_{R}^{12}\right)^{2}-\left(D_{R}^{13}\right)^{2} ; 0=\partial_{0} D_{R}^{10}+\partial_{1} D_{R}^{11}+\partial_{2} D_{R}^{12}+\partial_{3} D_{R}^{13}
$$

The conservative currents are $D_{R}^{1}, D_{R}^{8}$ and $D_{L}^{1}+D_{L}^{8}$. Next we get:

$$
\begin{aligned}
a_{1} \bar{a}_{1} & =4\left(\xi_{1}^{1} \bar{\eta}_{1}^{1}+\xi_{2}^{1} \bar{\eta}_{2}^{1}\right)\left(\bar{\xi}_{1}^{1} \eta_{1}^{1}+\bar{\xi}_{2}^{1} \eta_{2}^{1}\right)=4\left(\xi_{1}^{1} \bar{\xi}_{1}^{1} \eta_{1}^{1} \bar{\eta}_{1}^{1}+\xi_{1}^{1} \bar{\xi}_{2}^{1} \eta_{2}^{1} \bar{\eta}_{1}^{1}+\xi_{1}^{2} \bar{\xi}_{1}^{1} \eta_{1}^{1} \bar{\eta}_{2}^{1}+\xi_{2}^{1} \bar{\xi}_{2}^{1} \eta_{2}^{1} \bar{\eta}_{2}^{1}\right) \\
& =2\left(D_{R}^{10} D_{L}^{10}-D_{R}^{11} D_{L}^{11}-D_{R}^{12} D_{L}^{12}-D_{R}^{13} D_{L}^{13}\right)=2 D_{R}^{1} \cdot D_{L}^{1} .
\end{aligned}
$$

The same calculation gives for the other terms in $\rho^{2}$ :

$$
a_{2} \bar{a}_{2}=2 D_{L}^{1} \cdot D_{L}^{8} ; a_{3} \bar{a}_{3}=2 D_{R}^{1} \cdot D_{L}^{8} ; a_{4} \bar{a}_{4}=2 D_{L}^{1} \cdot D_{R}^{8} ; a_{5} \bar{a}_{5}=2 D_{R}^{1} \cdot D_{R}^{8} ; a_{6} \bar{a}_{6}=2 D_{R}^{8} \cdot D_{L}^{8} .
$$

This implies: 


$$
\begin{aligned}
\rho^{2} & \left.=2 D_{R}^{1} \cdot D_{L}^{1}+2 D_{L}^{1} \cdot D_{L}^{8}+2 D_{R}^{1} \cdot D_{L}^{8}+2 D_{L}^{1} \cdot D_{R}^{8}+2 D_{R}^{1} \cdot D_{L}^{8}+2 D_{R}^{8} \cdot D_{L}^{8}\right) \\
& =\left(D_{R}^{1}+D_{L}^{1}+D_{R}^{8}+D_{L}^{8}\right) \cdot\left(D_{R}^{1}+D_{L}^{1}+D_{R}^{8}+D_{L}^{8}\right)=\left\|J_{l}\right\|^{2} \\
\rho & =\left\|J_{l}\right\| .
\end{aligned}
$$

The space-time vector $v=J_{l} / \rho$ in the mass terms of the wave equations is then unitary and the wave Equation (1.19) read:

$$
\begin{aligned}
& 0=(\hat{\nabla}+2 i \hat{\mathrm{b}}+i m \hat{\mathrm{v}}) R^{1} . \\
& 0=\left(\bar{\nabla}-i\left(\frac{1}{2}+k\right) \hat{\mathrm{b}}+i m \hat{\mathrm{v}}\right) \tilde{R}^{8} . \\
& 0=\left[\nabla+i\left(\mathrm{~b}+3 w^{3}\right)+i m \mathrm{v}\right] \hat{L}^{1} . \\
& 0=\left[\tilde{\nabla}+i\left(\mathrm{~b}-3 w^{3}\right)+i m \mathrm{v}\right] \vec{L}^{8} .
\end{aligned}
$$

The unitary space-time vector $\mathrm{v}$ is similar to a reduced velocity and we name this vector "local reduced velocity". It is impossible to suppress this term: the replacement of $\mathrm{v}$ by 1 should suppress the crossing between the left and right terms in the Dirac equation, and this crossing is necessary to get the results of the Dirac equation for the electron. L. de Broglie often said that the wave equation of the electron is essentially relativistic: since the chiral currents are isotropic, none non-relativistic approximation can actually conserve the chiral properties of the lepton wave, and this is exactly the reason allowing us to say that the quantum wave of the electron can never be a solution of the Schrödinger equation, even at very small or null usual velocity. The use in quantum electrodynamics of a Schrödinger equation for any quantum state is the same kind of error than the use of a non-relativistic theory for the Maxwell laws of the electromagnetism: this automatically should suppress most of the physical properties of the electromagnetism (induction, electromagnetic waves and so on). An electron without spin does not exist.

\subsection{The Quark Sector}

We start from the wave equations obtained in [1], Equations (3.60)-(3.62) and (3.66)(3.69), with $n=2$ (red color). The other equations are similar, up to a circular permutation of $n=2,3,4$. This gives the system:

$$
\begin{aligned}
i \hat{\nabla} R^{2}= & \frac{2}{3} \hat{\mathrm{b}} R^{2}+\left(\hat{h}_{1}^{1}+i \hat{h}_{1}^{2}\right) R^{3}+\left(\hat{h}_{3}^{1}-i \hat{h}_{3}^{2}\right) R^{4}+\left(\hat{h}_{1}^{3}-\hat{h}_{3}^{3}\right) R^{2} \\
& +\frac{m_{q}}{\rho}\left(d_{2} \hat{L}^{2}-s_{5}^{23} \hat{R}^{3} \sigma_{1}+s_{3}^{23} \hat{L}^{3}+s_{5}^{42} \hat{R}^{4} \sigma_{1}+s_{4}^{42} \hat{L}^{4}\right. \\
& \left.+s_{5}^{52} \bar{R}^{6} \sigma_{1}+s_{5}^{72} \bar{R}^{7} \sigma_{1}+s_{4}^{52} \overline{L^{5}}+s_{4}^{62} \bar{L}^{6}+s_{4}^{72} \overline{L^{7}}\right) \\
i \bar{\nabla} \tilde{R}^{5}=- & \frac{4}{3} \hat{\mathrm{b}} \tilde{R}^{5}+\left(\hat{h}_{1}^{1}+i \hat{h}_{1}^{2}\right) \tilde{R}^{6}+\left(\hat{h}_{3}^{1}-i \hat{h}_{3}^{2}\right) \tilde{R}^{7}+\left(\hat{h}_{1}^{3}-\hat{h}_{3}^{3}\right) \tilde{R}^{5} \\
+ & \frac{m_{q}}{\rho}\left(d_{5} \bar{L}^{5}-s_{5}^{56} \bar{R}^{6} \sigma_{1}+s_{3}^{56} \bar{L}^{6}+s_{5}^{75} \bar{R}^{7} \sigma_{1}+s_{4}^{75} \overline{L^{7}}-s_{5}^{52} \hat{R}^{2} \sigma_{1}\right. \\
& \left.-s_{5}^{53} \hat{R}^{3} \sigma_{1}-s_{5}^{54} \hat{R}^{4} \sigma_{1}+s_{3}^{52} \hat{L}^{2}+s_{3}^{53} \hat{L}^{3}+s_{3}^{54} \hat{L}^{4}\right)
\end{aligned}
$$




$$
\begin{aligned}
-i \nabla \hat{L}^{2}= & \frac{\mathrm{b}}{3} \hat{L}^{2}+\left(w_{2}^{1}+i w_{2}^{2}\right) \bar{L}^{5}-w_{2}^{3} \hat{L}^{2}+\left(h_{1}^{1}+i h_{1}^{2}\right) \hat{L}^{3}+\left(h_{3}^{1}-i h_{3}^{2}\right) \hat{L}^{4}+\left(h_{1}^{3}-h_{3}^{3}\right) \hat{L}^{2} \\
& -\frac{m_{q}}{\rho}\left(\bar{d}_{2} R^{2}+\bar{s}_{2}^{23} L^{3} \sigma_{1}+\bar{s}_{4}^{23} R^{3}-\bar{s}_{2}^{42} L^{4} \sigma_{1}+\bar{s}_{3}^{42} R^{4}-\bar{s}_{2}^{52} \tilde{L}^{5} \sigma_{1}-\bar{s}_{2}^{62} \tilde{L}^{6} \sigma_{1}-\bar{s}_{2}^{72} \tilde{L}^{7} \sigma_{1}+\bar{s}_{3}^{52} \tilde{R}^{5}+\bar{s}_{3}^{62} \tilde{R}^{6}+\bar{s}_{3}^{72} \tilde{R}^{7}\right) . \\
-i \tilde{\nabla} \overline{L^{5}}= & \frac{\mathrm{b}}{3} \bar{L}^{5}+\left(w_{2}^{1}-i w_{2}^{2}\right) \tilde{L}^{2}+w_{2}^{3} \bar{L}^{5}+\left(h_{1}^{1}+i h_{1}^{2}\right) \bar{L}^{6}+\left(h_{3}^{1}-i h_{3}^{2}\right) \bar{L}^{4}+\left(h_{1}^{3}-h_{3}^{3}\right) \bar{L}^{5} \\
& -\frac{m_{q}}{\rho}\left(\bar{d}_{5} \tilde{R}^{5}+\bar{s}_{2}^{56} \tilde{L}^{6} \sigma_{1}+\bar{s}_{4}^{56} \tilde{R}^{6}-\bar{s}_{2}^{75} \tilde{L}^{7} \sigma_{1}+\bar{s}_{3}^{75} \tilde{R}^{7}+\bar{s}_{2}^{52} L^{2} \sigma_{1}+\bar{s}_{2}^{53} L^{3} \sigma_{1}+\bar{s}_{2}^{54} L^{4} \sigma_{1}+\bar{s}_{4}^{52} R^{2}+\bar{s}_{4}^{53} R^{3}+\bar{s}_{4}^{54} R^{4}\right) .
\end{aligned}
$$

The mass term $m_{q}$ may be different from the mass term $m$ of the lepton wave. The conservative $J_{q}$ current, which is the current of probability in the quark case, satisfies:

$$
J_{q}=D_{R}^{2}+D_{R}^{3}+D_{R}^{4}+D_{L}^{2}+D_{L}^{3}+D_{L}^{4}+D_{R}^{5}+D_{R}^{6}+D_{R}^{7}+D_{L}^{5}+D_{L}^{6}+D_{L}^{7} .
$$

Like in the previous section the calculation of $\rho$ gives:

$$
\rho=\left\|J_{q}\right\| ; \quad \mathrm{v}=\frac{J_{q}}{\rho} ; \quad\|\mathrm{v}\|=1 .
$$

The weak potentials satisfy:

$$
\begin{aligned}
& w_{2}^{1}=q D_{L}^{25}=q\left(L^{2} L^{5}+\tilde{L}^{5} \tilde{L}^{2}\right) ; w_{2}^{2}=q d_{L}^{25}=q\left(i L^{2} L^{5}-i \tilde{L}^{5} \tilde{L}^{2}\right) ; w_{2}^{3}=q\left(-D_{L}^{2}+D_{L}^{5}\right) ; \\
& w_{3}^{1}=q D_{L}^{36}=q\left(L^{3} L^{6}+\tilde{L}^{6} \tilde{L}^{3}\right) ; w_{3}^{2}=q d_{L}^{36}=q\left(i L^{3} L^{6}-i \tilde{L}^{6} \tilde{L}^{3}\right) ; w_{3}^{3}=q\left(-D_{L}^{3}+D_{L}^{6}\right) ; \\
& w_{4}^{1}=q D_{L}^{47}=q\left(L^{4} L^{7}+\tilde{L}^{7} \tilde{L}^{5}\right) ; w_{4}^{2}=q d_{L}^{47}=q\left(i L^{4} L^{7}-i \tilde{L}^{7} \tilde{L}^{4}\right) ; w_{4}^{3}=q\left(-D_{L}^{4}+D_{L}^{7}\right) .
\end{aligned}
$$

A calculation similar to (1.14) gives:

$$
\begin{gathered}
i \hat{\nabla} R^{2}=\frac{2}{3} \hat{\mathrm{b}} R^{2}+\left(\hat{h}_{1}^{1}+i \hat{h}_{1}^{2}\right) R^{3}+\left(\hat{h}_{3}^{1}-i \hat{h}_{3}^{2}\right) R^{4}+\left(\hat{h}_{1}^{3}-\hat{h}_{3}^{3}\right) R^{2}+m_{q} \hat{\mathrm{v}} R^{2} . \\
i \bar{\nabla} \tilde{R}^{5}=-\frac{4}{3} \hat{\mathrm{b}} \tilde{R}^{5}+\left(\hat{h}_{1}^{1}+i \hat{h}_{1}^{2}\right) \tilde{R}^{6}+\left(\hat{h}_{3}^{1}-i \hat{h}_{3}^{2}\right) \tilde{R}^{7}+\left(\hat{h}_{1}^{3}-\hat{h}_{3}^{3}\right) \tilde{R}^{5}+m_{q} \hat{\mathrm{v}} \tilde{R}^{5} . \\
-i \nabla \hat{L}^{2}=\frac{\mathrm{b}}{3} \hat{L}^{2}+\left(w_{2}^{1}+i w_{2}^{2}\right) \bar{L}^{5}-w_{2}^{3} \hat{L}^{2}+\left(h_{1}^{1}+i h_{1}^{2}\right) \hat{L}^{3}+\left(h_{3}^{1}-i h_{3}^{2}\right) \hat{L}^{4}+\left(h_{1}^{3}-h_{3}^{3}\right) \hat{L}^{2}-m_{q} \mathrm{v} \hat{L}^{2} . \\
-i \tilde{\nabla} \overline{L^{5}}=\frac{\mathrm{b}}{3} \overline{L^{5}}+\left(w_{2}^{1}-i w_{2}^{2}\right) \hat{L}^{2}+w_{2}^{3} \overline{L^{5}}+\left(h_{1}^{1}+i h_{1}^{2}\right) \bar{L}^{6}+\left(h_{3}^{1}-i h_{3}^{2}\right) \bar{L}^{4}+\left(h_{1}^{3}-h_{3}^{3}\right) \overline{L^{5}}-m_{q} \mathrm{v} \overline{L^{5}}
\end{gathered}
$$

The gauge bosons acting in (1.34) on the right part of the quark $d$ are made of only the right colored $d$ waves:

$$
\begin{aligned}
& h_{1}^{1}=\frac{g_{3}}{2} D_{R}^{32}=\frac{g_{3}}{2}\left(R^{2} \tilde{R}^{3}+R^{3} \tilde{R}^{2}\right) ; h_{1}^{2}=\frac{g_{3}}{2} d_{R}^{32}=\frac{g_{3}}{2}\left(-i R^{2} \tilde{R}^{3}+i R^{3} \tilde{R}^{2}\right) ; \\
& h_{1}^{3}=\frac{g_{3}}{2}\left(-D_{R}^{2}+D_{R}^{3}\right), \\
& h_{2}^{1}=\frac{g_{3}}{2} D_{R}^{43}=\frac{g_{3}}{2}\left(R^{3} \tilde{R}^{4}+R^{4} \tilde{R}^{3}\right) ; h_{2}^{2}=\frac{g_{3}}{2} d_{R}^{43}=\frac{g_{3}}{2}\left(-i R^{3} \tilde{R}^{4}+i R^{4} \tilde{R}^{3}\right) ; \\
& h_{2}^{3}=\frac{g_{3}}{2}\left(-D_{R}^{3}+D_{R}^{4}\right), \\
& h_{3}^{1}=\frac{g_{3}}{2} D_{R}^{24}=\frac{g_{3}}{2}\left(R^{4} \tilde{R}^{2}+R^{2} \tilde{R}^{4}\right) ; h_{3}^{2}=\frac{g_{3}}{2} d_{R}^{24}=\frac{g_{3}}{2}\left(-i R^{4} \tilde{R}^{2}+i R^{2} \tilde{R}^{4}\right) ; \\
& h_{3}^{3}=\frac{g_{3}}{2}\left(-D_{R}^{4}+D_{R}^{2}\right) .
\end{aligned}
$$


This gives:

$$
\left(\hat{h}_{1}^{1}+i \hat{h}_{1}^{2}\right) R^{3}+\left(\hat{h}_{3}^{1}-i \hat{h}_{3}^{2}\right) R^{4}=-2\left(\hat{h}_{1}^{3}-\hat{h}_{3}^{3}\right) R^{2}=-g_{3} \hat{J}_{d R} R^{2} ; J_{d R}=D_{R}^{2}+D_{R}^{3}+D_{R}^{4},
$$

then the wave Equation (1.34) of the right red $d$ quark is reduced to :

$$
0=\left(-i \hat{\nabla}+\frac{2}{3} \hat{\mathrm{b}}-\frac{g_{3}}{2} \hat{J}_{d R}+m_{q} \hat{\mathrm{v}}\right) R^{2} .
$$

Similarly the wave equations of the green and blue colors of the right $d$ quark are:

$$
\begin{aligned}
& 0=\left(-i \hat{\nabla}+\frac{2}{3} \hat{\mathrm{b}}-\frac{g_{3}}{2} \hat{J}_{d R}+m_{q} \hat{\mathrm{v}}\right) R^{3}, \\
& 0=\left(-i \hat{\nabla}+\frac{2}{3} \hat{\mathrm{b}}-\frac{g_{3}}{2} \hat{J}_{d R}+m_{q} \hat{\mathrm{v}}\right) R^{4} .
\end{aligned}
$$

The gauge bosons acting in (1.35) on the right part of the quark $u$ are made of only the right colored $u$ waves:

$$
\begin{aligned}
& h_{1}^{1}=\frac{g_{3}}{2} D_{R}^{56}=\frac{g_{3}}{2}\left(\tilde{R}^{5} R^{6}+\tilde{R}^{6} R^{5}\right) ; h_{1}^{2}=\frac{g_{3}}{2} d_{R}^{65}=\frac{g_{3}}{2}\left(-i \tilde{R}^{5} R^{6}+i \tilde{R}^{6} R^{5}\right) ; \\
& h_{1}^{3}=\frac{g_{3}}{2}\left(-D_{R}^{5}+D_{R}^{6}\right), \\
& h_{2}^{1}=\frac{g_{3}}{2} D_{R}^{67}=\frac{g_{3}}{2}\left(\tilde{R}^{6} R^{7}+\tilde{R}^{7} R^{6}\right) ; h_{2}^{2}=\frac{g_{3}}{2} d_{R}^{76}=\frac{g_{3}}{2}\left(-i \tilde{R}^{6} R^{7}+i \tilde{R}^{7} R^{6}\right) ; \\
& h_{2}^{3}=\frac{g_{3}}{2}\left(-D_{R}^{6}+D_{R}^{7}\right), \\
& h_{3}^{1}=\frac{g_{3}}{2} D_{R}^{75}=\frac{g_{3}}{2}\left(\tilde{R}^{7} R^{5}+\tilde{R}^{5} R^{7}\right) ; h_{3}^{2}=\frac{g_{3}}{2} d_{R}^{57}=\frac{g_{3}}{2}\left(-i \tilde{R}^{7} R^{5}+i \tilde{R}^{5} R^{7}\right) ; \\
& h_{3}^{3}=\frac{g_{3}}{2}\left(-D_{R}^{7}+D_{R}^{5}\right) .
\end{aligned}
$$

This gives:

$$
\left(\hat{h}_{1}^{1}+i \hat{h}_{1}^{2}\right) \tilde{R}^{6}+\left(\hat{h}_{3}^{1}-i \hat{h}_{3}^{2}\right) \tilde{R}^{7}=-2\left(\hat{h}_{1}^{3}-\hat{h}_{3}^{3}\right) \tilde{R}^{5}=-g_{3} \hat{J}_{u R} ; J_{u R}=D_{R}^{5}+D_{R}^{6}+D_{R}^{7},
$$

then the wave Equation (1.35) of the right red $u$ quark is reduced to :

$$
0=\left(-i \bar{\nabla}-\frac{4}{3} \hat{\mathrm{b}}-\frac{g_{3}}{2} \hat{J}_{u R}+m_{q} \hat{\mathrm{v}}\right) \tilde{R}^{5} .
$$

And the wave equations of the green and blue colors of the same right $u$ quark are:

$$
\begin{aligned}
& 0=\left(-i \bar{\nabla}-\frac{4}{3} \hat{\mathrm{b}}-\frac{g_{3}}{2} \hat{J}_{u R}+m_{q} \hat{\mathrm{v}}\right) \tilde{R}^{6}, \\
& 0=\left[-i \bar{\nabla}-\frac{4}{3} \hat{\mathrm{b}}-\frac{g_{3}}{2} \hat{J}_{u R}+m_{q} \hat{\mathrm{v}}\right) \tilde{R}^{7} .
\end{aligned}
$$

Next for the left waves we get a double dependence for the gauge bosons, because the weak potentials also change with the color and the strong potentials change with the savor. Similarly to (1.18) we have:

$$
\begin{aligned}
& \left(w_{2}^{1}+i w_{2}^{2}\right) \bar{L}^{5}=-2 q D_{L}^{5} \hat{L}^{2}=-2 w_{2}^{3} \hat{L}^{2} ;\left(w_{2}^{1}-i w_{2}^{2}\right) \hat{L}^{2}=-2 q D_{L}^{2} \overline{L^{5}}=2 w_{2}^{3} \overline{L^{5}} ; \\
& \left(w_{3}^{1}+i w_{3}^{2}\right) \bar{L}^{6}=-2 q D_{L}^{6} \hat{L}^{3}=-2 w_{3}^{3} \hat{L}^{3} ;\left(w_{3}^{1}-i w_{3}^{2}\right) \hat{L}^{3}=-2 q D_{L}^{3} \overline{L^{6}}=2 w_{3}^{3} \overline{L^{6}} ; \\
& \left(w_{4}^{1}+i w_{4}^{2}\right) \bar{L}^{7}=-2 q D_{L}^{7} \hat{L}^{4}=-2 w_{4}^{3} \hat{L}^{4} ;\left(w_{4}^{1}-i w_{4}^{2}\right) \hat{L}^{4}=-2 q D_{L}^{4} \bar{L}^{7}=2 w_{4}^{3} \overline{L^{7}} .
\end{aligned}
$$


The gauge bosons acting in (1.36) on the left part of the quark $\mathrm{d}$ are made of only the left colored d waves:

$$
\begin{aligned}
& h_{1}^{1}=\frac{g_{3}}{2} D_{L}^{23}=\frac{g_{3}}{2}\left(L^{2} \tilde{L}^{3}+L^{3} \tilde{L}^{2}\right) ; h_{1}^{2}=\frac{g_{3}}{2} d_{L}^{32}=\frac{g_{3}}{2}\left(-i L^{2} \tilde{L}^{3}+i L^{3} \tilde{L}^{2}\right) ; \\
& h_{1}^{3}=\frac{g_{3}}{2}\left(-D_{L}^{2}+D_{L}^{3}\right), \\
& h_{2}^{1}=\frac{g_{3}}{2} D_{L}^{34}=\frac{g_{3}}{2}\left(L^{3} \tilde{L}^{4}+L^{4} \tilde{L}^{3}\right) ; h_{2}^{2}=\frac{g_{3}}{2} d_{L}^{43}=\frac{g_{3}}{2}\left(-i L^{3} \tilde{L}^{4}+i L^{4} \tilde{L}^{3}\right) ; \\
& h_{2}^{3}=\frac{g_{3}}{2}\left(-D_{L}^{3}+D_{L}^{4}\right), \\
& h_{3}^{1}=\frac{g_{3}}{2} D_{L}^{42}=\frac{g_{3}}{2}\left(L^{4} \tilde{L}^{2}+L^{2} \tilde{L}^{4}\right) ; h_{3}^{2}=\frac{g_{3}}{2} d_{L}^{24}=\frac{g_{3}}{2}\left(-i L^{4} \tilde{L}^{2}+i L^{2} \tilde{L}^{4}\right) ; \\
& h_{3}^{3}=\frac{g_{3}}{2}\left(-D_{L}^{4}+D_{L}^{2}\right) .
\end{aligned}
$$

This gives:

$$
\left(h_{1}^{1}+i h_{1}^{2}\right) \hat{L}^{3}+\left(h_{3}^{1}-i h_{3}^{2}\right) \hat{L}^{4}=-g_{3} J_{d L} \hat{L}^{2} ; J_{d L}=D_{L}^{2}+D_{L}^{3}+D_{L}^{4},
$$

then the wave Equation (1.36) of the left red $d$ quark is reduced to :

$$
0=\left(i \nabla+\frac{\mathrm{b}}{3}-3 q D_{L}^{5}-\frac{g_{3}}{2} J_{d L}-m_{q} \mathrm{v}\right) \hat{L}^{2} .
$$

And the wave equations of the green and blue colors of the left $d$ quark are:

$$
\begin{aligned}
& 0=\left(i \nabla+\frac{\mathrm{b}}{3}-3 q D_{L}^{6}-\frac{g_{3}}{2} J_{d L}-m_{q} \mathrm{v}\right) \hat{L}^{3}, \\
& 0=\left(i \nabla+\frac{\mathrm{b}}{3}-3 q D_{L}^{7}-\frac{g_{3}}{2} J_{d L}-m_{q} \mathrm{v}\right) \hat{L}^{4} .
\end{aligned}
$$

The gauge bosons acting in (1.37) on the left part of the quark $u$ are made of only the left colored $u$ waves:

$$
\begin{aligned}
& h_{1}^{1}=\frac{g_{3}}{2} D_{L}^{56}=\frac{g_{3}}{2}\left(\tilde{L}^{5} L^{6}+\tilde{L}^{6} L^{5}\right) ; h_{1}^{2}=\frac{g_{3}}{2} d_{L}^{65}=\frac{g_{3}}{2}\left(i \tilde{L}^{6} L^{5}-i \tilde{L}^{5} L^{6}\right) ; \\
& h_{1}^{3}=\frac{g_{3}}{2}\left(-D_{L}^{5}+D_{L}^{6}\right), \\
& h_{2}^{1}=\frac{g_{3}}{2} D_{L}^{67}=\frac{g_{3}}{2}\left(\tilde{L}^{6} L^{7}+\tilde{L}^{7} L^{6}\right) ; h_{2}^{2}=\frac{g_{3}}{2} d_{R}^{76}=\frac{g_{3}}{2}\left(i \tilde{L}^{7} L^{6}-i \tilde{L}^{6} L^{7}\right) ; \\
& h_{2}^{3}=\frac{g_{3}}{2}\left(-D_{L}^{6}+D_{L}^{7}\right), \\
& h_{3}^{1}=\frac{g_{3}}{2} D_{L}^{75}=\frac{g_{3}}{2}\left(\tilde{L}^{7} L^{5}+\tilde{L}^{5} L^{7}\right) ; h_{3}^{2}=\frac{g_{3}}{2} d_{L}^{57}=\frac{g_{3}}{2}\left(i \tilde{L}^{5} L^{7}-i \tilde{L}^{7} L^{5}\right) ; \\
& h_{3}^{3}=\frac{g_{3}}{2}\left(-D_{L}^{7}+D_{L}^{5}\right) .
\end{aligned}
$$

This gives:

$$
\left(h_{1}^{1}+i h_{1}^{2}\right) \bar{L}^{6}+\left(h_{3}^{1}-i h_{3}^{2}\right) \vec{L}=g_{3} J_{u L} \bar{L}^{5} ; J_{u L}=D_{L}^{5}+D_{L}^{6}+D_{L}^{7},
$$

then the wave Equation (1.37) of the left red d quark is reduced to: 


$$
0=\left(i \tilde{\nabla}+\frac{\mathrm{b}}{3}-3 q D_{L}^{2}-\frac{g_{3}}{2} J_{u L}-m_{q} \mathrm{v}\right) \bar{L}^{5}
$$

And the wave equations of the green and blue colors of the same d quark are:

$$
\begin{aligned}
& 0=\left(i \tilde{\nabla}+\frac{\mathrm{b}}{3}-3 q D_{L}^{3}-\frac{g_{3}}{2} J_{u L}-m_{q} \mathrm{v}\right) \bar{L}^{6}, \\
& 0=\left(i \tilde{\nabla}+\frac{\mathrm{b}}{3}-3 q D_{L}^{4}-\frac{g_{3}}{2} J_{u L}-m_{q} \mathrm{v}\right) \vec{L}^{7} .
\end{aligned}
$$

So we describe all particles of the first generation in the SM with 8 left spinors and 8 right spinors, then 16 spinors together, we have obtained 16 simplified wave equations: four in (1.19), three in (1.40)-(1.41), three in (1.44)-(1.45), three in (1.49)-(1.50), three in (1.53)-(1.54). Each wave equation is equivalent to four numeric partial differential equations, we have the true number of numeric equations for our $64=16 \times 4$ real functions of space and time.

\section{Covariant Derivative}

In the frame of General Relativity (GR) the space-time manifold has in each point of the manifold a tangent space-time with a variable space-time basis $\left(e_{0}, e_{1}, e_{2}, e_{3}\right)$. The local coordinates of an event $x$ in this basis read:

$$
x=x^{0} e_{0}+x^{1} e_{1}+x^{2} e_{2}+x^{3} e_{3}=x^{\mu} e_{\mu} ; x^{0}=c t ; g_{\mu \nu}=e_{\mu} \cdot e_{\nu} .
$$

This point of view comes not directly from the ideas of $A$. Einstein, based on the concept of invariance, but from the ideas of his professor of mathematics, Minkowski. Naturally D. Hestenes and most of his followers [24] [25] [26] [27] used the space-time algebra, replacing the basis $\left(e_{0}, e_{1}, e_{2}, e_{3}\right)$ by an orthonormal variable basis $\left(\gamma_{0}, \gamma_{1}, \gamma_{2}, \gamma_{3}\right)$. Nevertheless we developed another way for many reasons that we explained in the introduction of [16]. A posteriori the previous section gives a new reason for the choice of $\mathrm{Cl}_{3}$ as the true framework of relativistic quantum mechanics: since the potentials in (1.40), (1.44), (1.49) and (1.53) are built from the chiral waves $R^{n}$ and $L^{n}$ the wave equations of the right and left parts do not add to give a wave equation for $\phi^{n}=R^{n}+L^{n}$. Then the Clifford algebra of space-time is not the best framework to build the relativistic quantum model able to include both GR and SM.

V. Fock [28] revisited the construction of GR from a physical point of view. His starting point was the same used by Einstein: the invariance of the light velocity in any moving frame. Since light is an electromagnetic wave, Fock considered an electromagnetic wave front and imposed only to two moving frames the same law for such a wave front. His second point is the use of inertial frames of reference ([28] p. 15): "There exist frames of reference in which the equations of motion have a particularly simple form ; in a certain sense these are the most 'natural' frames of reference. They are the inertial frames in which the motion of a body is uniform and rectilinear, provided no forces act on it." This point of view is implicit in our text, such an inertial frame is always used in quantum physics. From the invariance of the light velocity in any inertial frame, Fock proved that the transformation linking the coordinates of an event is ne- 
cessary linear: $x^{\prime \mu}=D_{v}^{\mu} x^{\nu}$ and he proved next that the transformation is exactly what we use in (1.1): the transformation is necessary a dilation, with a scale factor. But Fock did not start from quantum mechanics, nor from the wave of a particle with spin $1 / 2$. It is very hard to go from the group of the dilations to the $\mathrm{Cl}_{3}^{*}$ group, because the natural homomorphism is from $\mathrm{Cl}_{3}^{*}$ into the group of the dilations. In spite of the knowledge of the expansion of the Universe which is simply a growing scale factor, Fock did not use this dilation. He only find a reason to neglect the ratio of his dilation, getting the Lorenz transformation that he was searching.

Fock emphasized also this: "The equations of the gravitational, or any other field, are partial differential equations, the solutions of which are unique only when initial, boundary or other equivalent conditions are given. The field equations and the boundary conditions are connected and the latter can in no way be considered less important than the former. But, in problems relating to the whole of space, the boundary conditions refer to distant regions and their formulation requires knowledge of space as a whole". Consequently Fock used a space uniform at infinity. This also is implicit in the point of view of the $\mathrm{Cl}_{3}$ algebra used here since we suppose in (1.1) the existence of an orthonormal fixed direct basis $\left(\sigma_{1}, \sigma_{2}, \sigma_{3}\right)$ that any observer moving with uniform movement of translation in comparison with other ones may use. The $\left(\sigma_{\mu}\right)$ basis used by the observer of $x$ is also used by the observer of $x^{\prime}$. We go from the coordinates $x^{\mu}$ of an event seen by an observer in his frame to the coordinates $x^{\prime \mu}$ of the same event seen by another moving observer by (1.1) which implies:

$$
\begin{aligned}
\operatorname{det}\left(x^{\prime}\right) & =\left(x^{\prime 0}\right)^{2}-\left(x^{\prime 1}\right)^{2}-\left(x^{\prime 2}\right)^{2}-\left(x^{\prime 3}\right)^{2}=\operatorname{det}(M x \tilde{M}) \\
& =\operatorname{det}(M) \operatorname{det}(x) \operatorname{det}(\tilde{M})=r^{2} \operatorname{det}(x) \\
& =r^{2}\left[\left(x^{0}\right)^{2}-\left(x^{1}\right)^{2}-\left(x^{2}\right)^{2}-\left(x^{3}\right)^{2}\right] ; \quad r=|\operatorname{det}(M)| .
\end{aligned}
$$

This is exactly the result of Fock: The wave equation of the electromagnetic front wave:

$$
\frac{1}{c^{2}}\left(\frac{\partial \omega}{\partial t}\right)^{2}-(\operatorname{grad} \omega)^{2}=0
$$

has the same form in two inertial frames only if:

$$
\left(x^{\prime 0}\right)^{2}-\left(x^{\prime 1}\right)^{2}-\left(x^{\prime 2}\right)^{2}-\left(x^{\prime 3}\right)^{2}=r^{2}\left[\left(x^{0}\right)^{2}-\left(x^{1}\right)^{2}-\left(x^{2}\right)^{2}-\left(x^{3}\right)^{2}\right]
$$

We explained in the first part of this work [1] section 4 how this dilation may be enlarged to the case where $M$ is not independent of $x$ and may change from a point to another one on the space-time manifold. The main difference with the tensorial frame of GR is the difference between contravariant vectors and covariant vectors that we encountered in the first section: The contravariant vectors transform as [1] (4.3)-(4.5) while the covariant vectors transform as [1] (4.6)-(4.8). Moreover for the spinor waves we have four other forms of semi-variance: $R^{1}, \hat{L}^{1}, R^{8}$ and $\hat{L}^{8}$ transform with the four kinds of representations of $\mathrm{Cl}_{3}^{*}$ :

$$
R^{1} \mapsto R^{\prime 1}=M R^{1} ; R^{8} \mapsto R^{\prime 8}=R^{8} M ; \hat{L}^{1} \mapsto \hat{L}^{\prime 1}=\hat{M} \hat{L}^{1} ; \hat{L}^{8} \mapsto \hat{L}^{\prime 8}=\hat{L^{8}} \hat{M}
$$


We then have four different forms of gradient applying into the wave equations:

$$
\boldsymbol{D}=\nabla-\left(\nabla \hat{M}^{-1}\right) \hat{M} ; \hat{\mathbf{D}} ; \tilde{\mathbf{D}} ; \overline{\mathbf{D}} \text {. }
$$

This appeared in the wave equations (1.6)-(1.9) and (1.27)-(1.30). Even in the case of a flat space with null Christoffel symbols we get again these four kinds of semi-variance, because the $i=\sigma_{1} \sigma_{2} \sigma_{3}$ which orients the space is in the kernel of the homomorphism $M \mapsto D$ :

$$
\boldsymbol{D}=\nabla-i \frac{\mathrm{b}}{2} ; \nabla=\boldsymbol{D}+i \frac{\mathrm{b}}{2} ; \hat{\nabla}=\hat{\boldsymbol{D}}-i \frac{\hat{\mathrm{b}}}{2} ; \tilde{\nabla}=\tilde{\boldsymbol{D}}-i \frac{\mathrm{b}}{2} ; \bar{\nabla}=\overline{\boldsymbol{D}}+i \frac{\hat{\mathrm{b}}}{2}
$$

Then the wave equations equivalent to (1.19) read:

$$
\begin{aligned}
& 0=\left(-i \hat{\mathbf{D}}+\frac{3}{2} \hat{\mathrm{b}}+m \hat{\mathrm{v}}\right) R^{1}, \\
& 0=(-i \overline{\mathbf{D}}-k \hat{\mathrm{b}}+m \hat{\mathrm{v}}) \tilde{R}^{8}, \\
& 0=\left(-i \boldsymbol{D}+\frac{3}{2} \mathrm{~b}+3 w^{3}+m \mathrm{v}\right) \hat{L}^{1}, \\
& 0=\left(-i \tilde{\mathbf{D}}+\frac{\mathrm{b}}{2}-3 w^{3}+m \mathrm{v}\right) \vec{L}^{8} .
\end{aligned}
$$

Similarly the wave equations of the colored quarks read, with $n=2,3,4$ :

$$
\begin{aligned}
& 0=\left(-i \hat{\mathbf{D}}+\frac{\hat{\mathrm{b}}}{6}-\frac{g_{3}}{2} \hat{J}_{d R}+m_{q} \hat{\mathrm{v}}\right) R^{n}, \\
& 0=\left(-i \overline{\boldsymbol{D}}-\frac{5}{6} \hat{\mathrm{b}}-\frac{g_{3}}{2} \hat{J}_{u R}+m_{q} \hat{\mathrm{v}}\right) \tilde{R}^{3+n}, \\
& 0=\left(-i \boldsymbol{D}+\frac{\mathrm{b}}{6}+3 q D_{L}^{3+n}+\frac{g_{3}}{2} J_{d L}+m_{q} \mathrm{v}\right) \hat{L}^{n}, \\
& 0=\left(-i \tilde{\mathbf{D}}-\frac{5}{6} \mathrm{~b}+3 q D_{L}^{n}+\frac{g_{3}}{2} J_{u L}+m_{q} \mathrm{v}\right) \bar{L}^{3+n} .
\end{aligned}
$$

\section{Relativistic Invariance}

The $S L(2, \mathbb{C})$ group used in relativistic quantum mechanics to account for the spin $1 / 2$ of the electron is a subgroup of the $G L(2, \mathbb{C})=C l_{3}^{*}$ group which is the natural geometric group of invariance with the 3-dimensional space. Under the dilation generated by any $M$ element of $C l_{3}^{*}$ satisfying (1.1) and (2.2) we have (see [16] 2.2.1):

$$
\begin{aligned}
& \boldsymbol{D}=\bar{M} \mathbf{D}^{\prime} \hat{M} ; \hat{\mathbf{D}}=\tilde{M} \hat{\mathbf{D}}^{\prime} M ; \tilde{\mathbf{D}}=\bar{M} \tilde{\mathbf{D}}^{\prime} \hat{M} ; \overline{\mathbf{D}}=\tilde{M} \overline{\mathbf{D}}^{\prime} M \\
& m=m^{\prime} r ; \rho^{\prime}=r \rho ; M \bar{M}=r e^{i \theta} ; \hat{M} \tilde{M}=r e^{-i \theta} ; J_{l}^{\prime}=M J_{l} \tilde{M} ; J_{l}=M^{-1} J^{\prime} \hat{M}^{-1} .
\end{aligned}
$$

We then get:

$$
\frac{m}{\rho} \hat{J}_{l} R^{1}=\frac{m^{\prime} r^{2}}{\rho^{\prime}} \tilde{M} \tilde{M}^{-1} \hat{M}^{-1} \hat{J}_{l}^{\prime} \bar{M}^{-1} M^{-1} M R^{1}=\frac{m^{\prime}}{\rho^{\prime}} r^{2} \tilde{M} r^{-1} \mathrm{e}^{i \theta} \hat{J}_{l}^{\prime} r^{-1} \mathrm{e}^{-i \theta} R^{\prime 1}=\frac{m^{\prime}}{\rho^{\prime}} \tilde{M} \hat{J}_{l}^{\prime} R^{\prime 1} .
$$

This gives:

$$
0=\left(-i \hat{\boldsymbol{D}}+\frac{3}{2} \hat{\mathrm{b}}+\frac{m}{\rho} \hat{J}_{l}\right) R^{1}=\tilde{M}\left(-i \hat{\boldsymbol{D}}^{\prime}+\frac{3}{2} \hat{\mathrm{b}}^{\prime}+\frac{m^{\prime}}{\rho^{\prime}} \hat{J}_{l}^{\prime} R^{\prime 1}\right)
$$


Since $M$ is invertible we have the equivalence:

$$
0=\left(-i \hat{\boldsymbol{D}}+\frac{3}{2} \hat{\mathrm{b}}+\frac{m}{\rho} \hat{J}_{l}\right) R^{1} \Leftrightarrow 0=\left(-i \hat{\boldsymbol{D}}^{\prime}+\frac{3}{2} \hat{\mathrm{b}}^{\prime}+\frac{m^{\prime}}{\rho^{\prime}} \hat{J}_{l}^{\prime}\right) R^{\prime 1}
$$

And it is the same for (2.8)-(2.9). It is this form invariance that is named "relativistic invariance" in the Dirac theory.

\section{The Electromagnetic Field}

In the SM the electromagnetic field is a field of bivectors (antisymmetric second rank tensor) on the relativistic point of view, and a field of operators on the quantum point of view. The motion of this field comes from a special part of the Lagrangian density. We cannot use this way, because we do not believe into a meta-physical necessity of the Lagrangian mechanism for any physical field. The Lagrangian density comes from the real part of the invariant form of the wave equations. Consequently only the wave equations of the fermion part of the SM are compulsory and all must come from them. We know that the particles of this electromagnetic field, the photons, are bosons with no mass. And we shall prove that this is the case for:

$$
A=\sum_{n=1}^{8}\left(D_{R}^{n}-D_{L}^{n}\right)
$$

We have explained in [23] why the derivative of a vector current like $D_{L}^{1}$ must be defined by:

$$
d^{-}\left(D_{L}^{1}\right)=\left(\nabla \hat{L}^{1}\right) \hat{L}^{1}-L^{1}\left(\tilde{L}^{1} \hat{\nabla}\right)
$$

Now the covariant derivative is more general than the partial derivative, so we replace this by:

$$
D^{-}\left(D_{L}^{1}\right)=\left(\boldsymbol{D} \hat{L}^{1}\right) \bar{L}^{1}-L^{1}\left(\tilde{L}^{1} \hat{\mathbf{D}}\right) ; \quad F=\sum_{n=1}^{8}\left[D^{-}\left(D_{R}^{n}\right)-D^{-}\left(D_{L}^{n}\right)\right] .
$$

This derivative will be generalized in a further article. This kind of derivative is sufficient to avoid the infinity of tensorial densities coming from the use of simple partial derivatives in the Dirac theory [3]. This derivative gives a field of bivectors with value in $\mathrm{Cl}_{3}$ that may be thought of as a field of operators acting on this field itself. We get, in the dilation generated by any $M$ element and with (1.4): $F^{\prime}=M F M^{-1}$ and this relation is compulsory because compatible with the product of operators. Equations (2.8) gives:

$$
\begin{aligned}
& \boldsymbol{D} \hat{R}^{1}=i\left(\frac{3}{2} \mathrm{~b}+m \mathrm{v}\right) \hat{R}^{1} ; \quad \tilde{R}^{1} \overline{\mathbf{D}}=i \tilde{R}^{1}\left(\frac{3}{2} \hat{\mathrm{b}}+m \hat{\mathrm{v}}\right) ; \\
& \tilde{\mathbf{D}} \bar{R}^{8}=i(-k \mathrm{~b}+m \mathrm{v}) \bar{R}^{8} ; R^{8} \hat{\mathbf{D}}=i R^{8}(-k \hat{\mathrm{b}}+m \hat{\mathrm{v}}) ; \\
& \boldsymbol{D} \hat{L}^{1}=-i\left(\frac{3}{2} \mathrm{~b}+3 w^{3}+m \mathrm{v}\right) \hat{L}^{1} ; \quad \tilde{L}^{1} \overline{\mathbf{D}}=-i \tilde{L}^{1}\left(\frac{3}{2} \hat{\mathrm{b}}+3 \hat{w}^{3}+m \hat{\mathrm{v}}\right) ; \\
& \tilde{\mathbf{D}} \bar{L}^{8}=-i\left(\frac{1}{2} \mathrm{~b}-3 w^{3}+m \mathrm{v}\right) \bar{L}^{8} ; L^{8} \hat{\mathbf{D}}=-i L^{8}\left(\frac{1}{2} \hat{\mathrm{b}}-3 \hat{w}^{3}+m \hat{\mathrm{v}}\right) .
\end{aligned}
$$


We get with $2 A \wedge B=A \hat{B}-B \hat{A}$ :

$$
\begin{aligned}
& D^{-}\left(D_{R}^{1}\right)=\left(\boldsymbol{D} \hat{R}^{1}\right) \bar{R}^{1}-R^{1}\left(\tilde{R}^{1} \overline{\boldsymbol{D}}\right)=2 i\left(\frac{3}{2} \mathrm{~b}+m \mathrm{v}\right) \wedge D_{R}^{1}, \\
& D^{-}\left(D_{R}^{8}\right)=\left(\tilde{\boldsymbol{D}} \bar{R}^{8}\right) \hat{R}^{8}-\tilde{R}^{8}\left(R^{8} \hat{\boldsymbol{D}}\right)=2 i(-k \mathrm{~b}+m \mathrm{v}) \wedge D_{R}^{8}, \\
& D^{-}\left(D_{L}^{1}\right)=\left(\boldsymbol{D} \hat{L}^{1}\right) \bar{L}^{1}-L^{1}\left(\tilde{L}^{1} \overline{\boldsymbol{D}}\right)=-2 i\left(\frac{3}{2} \mathrm{~b}+3 w^{3}+m \mathrm{v}\right) \wedge D_{L}^{1}, \\
& D^{-}\left(D_{L}^{8}\right)=\left(\tilde{\boldsymbol{D}} \bar{L}^{8}\right) \hat{L}^{8}-\tilde{L}^{8}\left(L^{8} \hat{\boldsymbol{D}}\right)=-2 i\left(\frac{1}{2} \mathrm{~b}-3 w^{3}+m \mathrm{v}\right) \wedge D_{L}^{8} .
\end{aligned}
$$

We then get for the electromagnetic field created by the lepton wave:

$$
\begin{aligned}
F_{l}= & D^{-}\left(A_{l}\right)=D^{-}\left(D_{R}^{1}\right)+D^{-}\left(D_{R}^{8}\right)-D^{-}\left(D_{L}^{1}\right)-D^{-}\left(D_{L}^{8}\right) \\
= & 2 i\left[\mathrm{~b} \wedge\left(\frac{3}{2} D_{R}^{1}+\frac{3}{2} D_{L}^{1}+\frac{1}{2} D_{L}^{8}-k D_{R}^{8}\right)\right. \\
& \left.+3 w^{3} \wedge\left(D_{L}^{1}-D_{L}^{8}\right)+\frac{m}{\rho} J_{l} \wedge\left(D_{R}^{1}+D_{R}^{8}+D_{L}^{1}+D_{L}^{8}\right)\right] .
\end{aligned}
$$

These last terms cancel with (1.12) and (1.17) and we get:

$$
F_{l}=2 i \mathrm{~b} \wedge\left(\frac{3}{2} D_{R}^{1}+\frac{3}{2} D_{L}^{1}+\frac{1}{2} D_{L}^{8}-k D_{R}^{8}\right) .
$$

Similarly the first wave Equation (2.9) gives:

$$
\begin{aligned}
& \boldsymbol{D} \hat{R}^{2}=i\left(\frac{1}{6} \mathrm{~b}-\frac{g_{3}}{2} J_{d R}+m_{q} \mathrm{v}\right) \hat{R}^{2}, \\
& D^{-}\left(D_{R}^{2}\right)=2 i\left(\frac{1}{6} \mathrm{~b}-\frac{g_{3}}{2} J_{d R}+m_{q} \mathrm{v}\right) \wedge D_{R}^{2}, \\
& D^{-}\left(D_{R}^{3}\right)=2 i\left(\frac{1}{6} \mathrm{~b}-\frac{g_{3}}{2} J_{d R}+m_{q} \mathrm{v}\right) \wedge D_{R}^{3}, \\
& D^{-}\left(D_{R}^{4}\right)=2 i\left(\frac{1}{6} \mathrm{~b}-\frac{g_{3}}{2} J_{d R}+m_{q} \mathrm{v}\right) \wedge D_{R}^{4}, \\
& D^{-}\left(D_{R}^{2}\right)+D^{-}\left(D_{R}^{3}\right)+D^{-}\left(D_{R}^{4}\right)=2 i\left(\frac{1}{6} \mathrm{~b}+m_{q} \mathrm{v}\right) \wedge J_{d R} .
\end{aligned}
$$

Next the second Equation (2.9) gives:

$$
\begin{aligned}
& \tilde{\mathbf{D}} \bar{R}^{5}=i\left(-\frac{5}{6} b-\frac{g_{3}}{2} J_{u R}+m_{q} \mathrm{v}\right) \bar{R}^{5} ; R^{5} \hat{\boldsymbol{D}}=i R^{5}\left(-\frac{5}{6} \hat{\mathrm{b}}-\frac{g_{3}}{2} \hat{J}_{u R}+m_{q} \hat{\mathrm{v}}\right), \\
& D^{-}\left(D_{R}^{5}\right)=\left(\tilde{\boldsymbol{D}} \bar{R}^{5}\right) \hat{R}^{5}-\tilde{R}^{5}\left(R^{5} \hat{\boldsymbol{D}}\right)=2 i\left(-\frac{5}{6} \mathrm{~b}-\frac{g_{3}}{2} J_{u R}+m_{q} \mathrm{v}\right) \wedge D_{R}^{5}, \\
& D^{-}\left(D_{R}^{6}\right)=2 i\left(-\frac{5}{6} \mathrm{~b}-\frac{g_{3}}{2} J_{u R}+m_{q} \mathrm{v}\right) \wedge D_{R}^{6}, \\
& D^{-}\left(D_{R}^{7}\right)=2 i\left(-\frac{5}{6} \mathrm{~b}-\frac{g_{3}}{2} J_{u R}+m_{q} \mathrm{v}\right) \wedge D_{R}^{7}, \\
& D^{-}\left(D_{R}^{5}\right)+D^{-}\left(D_{R}^{6}\right)+D^{-}\left(D_{R}^{7}\right)=2 i\left(-\frac{5}{6} \mathrm{~b}+m_{q} \mathrm{v}\right) \wedge J_{u R} .
\end{aligned}
$$


We then get:

$$
\sum_{n=2}^{7} D^{-}\left(D_{R}^{n}\right)=2 i\left[\frac{1}{6} \mathrm{~b} \wedge\left(J_{d R}-5 J_{u R}\right)+m_{q} \mathrm{v} \wedge\left(J_{d R}+J_{u R}\right)\right] .
$$

Next with the left waves and the third Equation (2.9) we get:

$$
\begin{aligned}
& \boldsymbol{D} \hat{L}^{2}=-i\left(\frac{\mathrm{b}}{6}+3 q D_{L}^{5}+\frac{g_{3}}{2} J_{d L}+m_{q} \mathrm{v}\right) \hat{L}^{2} ; \tilde{L}^{2} \overline{\mathbf{D}}=-i \tilde{L}^{2}\left(\frac{\hat{\mathrm{b}}}{6}+3 q \hat{D}_{L}^{5}+\frac{g_{3}}{2} \hat{J}_{d L}+m_{q} \hat{\mathrm{v}}\right) \\
& D^{-}\left(D_{L}^{2}\right)=\left(\boldsymbol{D} \hat{L}^{2}\right) \bar{L}^{2}-L^{2}\left(\tilde{L}^{2} \overline{\mathbf{D}}\right)=-2 i\left(\frac{b}{6}+3 q D_{L}^{5}+\frac{g_{3}}{2} J_{d L}+m_{q} \mathrm{v}\right) \wedge D_{L}^{2}, \\
& D^{-}\left(D_{L}^{3}\right)=-2 i\left(\frac{\mathrm{b}}{6}+3 q D_{L}^{6}+\frac{g_{3}}{2} J_{d L}+m_{q} \mathrm{v}\right) \wedge D_{L}^{3}, \\
& D^{-}\left(D_{L}^{4}\right)=-2 i\left(\frac{\mathrm{b}}{6}+3 q D_{L}^{7}+\frac{g_{3}}{2} J_{d L}+m_{q} \mathrm{v}\right) \wedge D_{L}^{4}, \\
& D^{-}\left(D_{L}^{2}\right)+D^{-}\left(D_{L}^{3}\right)+D^{-}\left(D_{L}^{4}\right)=2 i\left(-\frac{\mathrm{b}}{6}\right) \wedge J_{d L}-6 i q\left(D_{L}^{5} \wedge D_{L}^{2}+D_{L}^{6} \wedge D_{L}^{3}+D_{L}^{7} \wedge D_{L}^{4}\right) \\
& \quad-2 i m_{q} \mathrm{v} \wedge J_{d L} \cdot
\end{aligned}
$$

Next with the last Equation (2.9) we get:

$$
\begin{aligned}
& \tilde{\boldsymbol{D}} \overline{L^{5}}=-i\left(-\frac{5 \mathrm{~b}}{6}+3 q D_{L}^{2}+\frac{g_{3}}{2} J_{u L}+m_{q} \mathrm{v}\right) \overline{L^{5}} ; L^{5} \hat{\boldsymbol{D}}=-i L^{5}\left(-\frac{5 \hat{\mathrm{b}}}{6}+3 q \hat{D}_{L}^{2}+\frac{g_{3}}{2} \hat{J}_{u L}+m_{q} \mathrm{v}\right) \\
& D^{-}\left(D_{L}^{5}\right)=\left(\tilde{\mathbf{D}} \overline{L^{5}}\right) \hat{L^{5}}-\tilde{L}^{5}\left(L^{5} \hat{\boldsymbol{D}}\right)=-2 i\left(-\frac{5 \mathrm{~b}}{6}+3 q D_{L}^{2}+\frac{g_{3}}{2} J_{u L}+m_{q} \mathrm{v}\right) \wedge D_{L}^{5}, \\
& D^{-}\left(D_{L}^{6}\right)=-2 i\left(-\frac{5 \mathrm{~b}}{6}+3 q D_{L}^{3}+\frac{g_{3}}{2} J_{u L}+m_{q} \mathrm{v}\right) \wedge D_{L}^{6}, \\
& D^{-}\left(D_{L}^{7}\right)=-2 i\left(-\frac{5 \mathrm{~b}}{6}+3 q D_{L}^{4}+\frac{g_{3}}{2} J_{u L}+m_{q} \mathrm{v}\right) \wedge D_{L}^{7}, \\
& D^{-}\left(D_{L}^{5}\right)+D^{-}\left(D_{L}^{6}\right)+D^{-}\left(D_{L}^{7}\right)=2 i \frac{5 \mathrm{~b}}{6} \wedge J_{u L}-6 i q\left(D_{L}^{2} \wedge D_{L}^{5}+D_{L}^{3} \wedge D_{L}^{6}+D_{L}^{4} \wedge D_{L}^{7}\right)
\end{aligned}
$$

We then get:

$$
\sum_{n=2}^{7} D^{-}\left(D_{L}^{n}\right)=2 i\left(-\frac{\mathrm{b}}{6} \wedge J_{d L}+\frac{5 \mathrm{~b}}{6} \wedge J_{u L}\right)-2 i m_{q} \mathrm{v} \wedge\left(J_{d L}+J_{u L}\right) .
$$

By subtracting this from (3.10) we get:

$$
\begin{aligned}
F_{q} & =D^{-}\left(A_{q}\right)=\sum_{n=2}^{7}\left[\left(D^{-}\left(D_{R}^{n}\right)-D^{-}\left(D_{L}^{n}\right)\right]\right. \\
& =\frac{i}{3} \mathrm{~b} \wedge\left(J_{d R}-5 J_{u R}+J_{d L}-5 J_{u L}\right)+2 i \frac{m_{q}}{\rho} J_{q} \wedge\left(J_{d R}+J_{u R}+J_{d L}+J_{u L}\right) .
\end{aligned}
$$

And $J_{q}=J_{d R}+J_{u R}+J_{d L}+J_{u L}$ : the last exterior product cancels, then the electromagnetic field loses the mass term. We get:

$$
F_{q}=\frac{i}{3} \mathrm{~b} \wedge\left(J_{d R}-5 J_{u R}+J_{d L}-5 J_{u L}\right) \text {. }
$$

The electromagnetic field $F=F_{l}+F_{q}$ has then no mass term. The previous calcula- 
tion shows that this peculiar cancellation does not occur for other gauge fields. This is the reason of our choice of $A$ as the gauge boson of the electric gauge, boson that is known as the photon in the SM. We must recall that in quantum mechanics the derived fields are second, only the potential space-time vectors take place in the wave equations.

\section{Pauli Principle}

Studying the anomalous Zeeman effect, Pauli found the necessity of the "spin number" of the electron in the frame of the Bohr's model of atom. And he came to his principle [29]: "There can never be two or more equivalent electrons in an atom, for which in strong fields the values of all quantum numbers $n, l, j, m_{j}$ are the same. If an electron is present in the atom, for which these quantum numbers have definite values, this state is occupied." This principle and the quantum wave of the electron are today the basis of the understanding of the periodic table of the chemical elements.

Since its formulation is made in the frame of the non-relativistic wave of a system of electrons we cannot follow the usual explanation of this principle by the anti-symmetrization of the wave. The only compulsory wave equation is the wave of one electron and more generally of one fermion. Moreover the explanation by the four quantum numbers $\boldsymbol{n}, l, j, m_{j}$ given by Pauli himself is not directly the explanation coming from the Dirac theory of the electron. The resolution of the Dirac equation in the case of the Coulombian potential gives two kinds of solutions, with a quantum number $\kappa= \pm 1, \pm 2, \pm 3 \cdots$ which gives $\boldsymbol{n}(\boldsymbol{n}+1)$ states if $\kappa>0$ and $\boldsymbol{n}(\boldsymbol{n}-1)$ states if $\boldsymbol{\kappa}<0$ [30]. The quantum number $\kappa$ comes from the separation in spherical coordinates of the radial and angular functions. The quantum number $m_{j}$ comes from the separation between the angular parameters, even if it is also the proper value of the operator $J^{3}$ [31]. The quantum number $l$ of the Bohr's theory is absent of the calculation. The quantum number $j$ of the total kinetic momentum is only indirectly present: $j=|\kappa|-\frac{1}{2}$. The other quantum numbers are the degrees of the Gegenbauer's polynomial giving the angular functions (the Legendre polynomials with degree $l$ of the non-relativistic calculation are only linear combinations of two Gegenbauer's polynomial of different degrees) and the degree of the Laguerre's polynomials giving the radial functions (see [16] Appendix C). These polynomials are obtained only when the condition of integrability of the wave is imposed. This condition comes from the necessity to normalize the wave which itself comes from the equivalence principle between inertial and gravitational mass-energy (see [16] 9.2): $T_{0}^{0}$ being the density of energy of the wave and $E$ the global energy of the electron we have in any inertial frame and for any stationary state $\hbar c T_{0}^{0}=E J^{0}$ and:

$$
\iiint \mathrm{d} v T_{0}^{0}=E \Leftrightarrow \iiint \mathrm{d} v \frac{J^{0}}{\hbar c}=1 .
$$

This last equality allows us to define a norm for the wave $\phi$ of the electron:

$$
\|\phi\|=\iiint \mathrm{d} v \frac{J^{0}}{\hbar c} ; \quad\|\phi\|=1 .
$$


Next the Dirac equation which is the linear approximation of our non-linear wave equation in the electron case is satisfied by the sum and the difference of two solutions. The previous norm defines a scalar product:

$$
\phi_{1} \cdot \phi_{2}=\frac{1}{4}\left(\left\|\phi_{1}+\phi_{2}\right\|^{2}-\left\|\phi_{1}-\phi_{2}\right\|^{2}\right) .
$$

With the kind of functions used by the separation of variables in spherical coordinates, the condition of orthogonality for this Euclidean scalar product is automatically satisfied by the different solutions. Moreover the condition of orthogonality for the Euclidean scalar product is exactly equivalent to the cancellation of the Hermitian scalar product of quantum mechanics [31]. The meaning of the Pauli principle is then: two electrons in an atom occupy orthogonal normalized states. The true origin of this condition is the principle of equivalence between inertial and gravitational mass-energy.

\section{The Multi-Photons Field}

The quantized electromagnetic field is described with operators of creation and annihilation: the operator of creation adds one photon to the electromagnetic field and the operator of annihilation suppresses one photon. We previously studied the wave of a unique fermion and the potential present in the wave equations is linked to this unique fermion. The dilation generated by any $M$ element satisfies (1.1), (1.4) and (2.10), then (3.3) gives:

$$
\begin{aligned}
D^{-}\left(D_{L}^{\prime 1}\right) & =\left(\boldsymbol{D}^{\prime} \hat{L}^{1}\right) \bar{L}^{1}-L^{1}\left(\tilde{L}^{\prime} \hat{\mathbf{D}}^{\prime}\right) \\
& =\left[\left(\bar{M}^{-1} \boldsymbol{D} \hat{M}^{-1}\right)\left[\left(\hat{M} \hat{L}^{1}\right)\right]\left(\overline{M L}^{1}\right)-\left(M L^{1}\right)\left(\tilde{M} L^{1}\right)\left(\tilde{M}^{-1} \hat{\mathbf{D}} M^{-1}\right)\right] \\
& =\bar{M}^{-1}\left(\boldsymbol{D} \hat{L}^{1}\right) \bar{L}^{1} \bar{M}^{1}-M L^{1}\left[\tilde{L}^{1} \tilde{M} \tilde{M}^{-1} \hat{\boldsymbol{D}}\right] M^{-1} .
\end{aligned}
$$

With $\bar{M} M=M \bar{M}=\operatorname{det}(M)=r \mathrm{e}^{\mathrm{i} \theta}$ we get:

$$
\begin{aligned}
& \bar{M}=r \mathrm{e}^{i \theta} M^{-1} ; \bar{M}^{-1}=r^{-1} \mathrm{e}^{-i \theta} M \\
& \bar{M}^{-1}\left(\boldsymbol{D} \hat{L}^{1}\right) \bar{L}^{1} \bar{M}=r^{-1} \mathrm{e}^{-i \theta} M\left(\boldsymbol{D} \hat{L}^{1}\right) \bar{L} \bar{M}=M\left(\boldsymbol{D} \hat{L^{1}}\right) \bar{L} \bar{M} r^{-1} \mathrm{e}^{-i \theta} \\
&=M\left(\boldsymbol{D} \hat{L}^{1}\right) \bar{L} \bar{M} \bar{M}^{-1} M^{-1}=M\left(\boldsymbol{D} \hat{L}^{1}\right) \bar{L} M^{-1}
\end{aligned}
$$

Then (5.1) gives:

$$
\begin{aligned}
D^{-}\left(D_{L}^{\prime 1}\right) & =M\left(\boldsymbol{D} \hat{L}^{1}\right) \vec{L}^{1} M^{-1}-M L^{1}\left(\tilde{L}^{1} \hat{\boldsymbol{D}}\right) M^{-1}=M\left[\left(\boldsymbol{D} \hat{L}^{1}\right) L^{1}-L^{1}\left(\tilde{L}^{1} \hat{\boldsymbol{D}}\right)\right] M^{-1} \\
& =M D^{-}\left(D_{L}^{1}\right) M^{-1}, \\
F^{\prime} & =\sum_{n=1}^{8}\left[D^{-}\left(D_{R}^{\prime n}\right)-D^{-}\left(D_{L}^{\prime n}\right)\right]=\sum_{n=1}^{8}\left[M D^{-}\left(D_{R}^{n}\right) M^{-1}-M D^{-}\left(D_{L}^{n}\right) M^{-1}\right], \\
F^{\prime} & =M F M^{-1} .
\end{aligned}
$$

Now if the field of a system of two photons $F_{(1)}$ and $F_{(2)}$ satisfies:

$$
F_{12}=\frac{1}{2}\left(F_{(1)} F_{(2)}+F_{(2)} F_{(1)}\right),
$$

this field also transforms like the field of a unique photon: 


$$
\begin{aligned}
F_{12}^{\prime} & =\frac{1}{2}\left(F_{(1)}^{\prime} F_{(2)}^{\prime}+F_{(2)}^{\prime} F_{(1)}^{\prime}\right)=\frac{1}{2}\left[\left(M F_{(1)} M^{-1}\right)\left(M F_{(2)} M^{-1}\right)+\left(M F_{(2)} M^{-1}\right)\left(M F_{(1)} M^{-1}\right)\right] \\
& =\frac{1}{2} M\left(F_{(1)} F_{(2)}+F_{(2)} F_{(1)}\right) M^{-1}=M F_{12} M^{-1} .
\end{aligned}
$$

This is generalized by using the group $S(n)$ of permutations:

$$
F_{1,2, \cdots, n}=\sum_{\sigma \in S(n)} \frac{1}{n !} F_{\sigma(1)} F_{\sigma(2)} \cdots F_{\sigma(n)} ; F_{1,2, \cdots, n}^{\prime}=M F_{1,2, \cdots, n} M^{-1} .
$$

The quantized electromagnetic field is the general element $\mathcal{F}$ of the linear space generated by all these fields of systems of photons, satisfying again:

$$
\mathcal{F}^{\prime}=M \mathcal{F} M^{-1} \text {. }
$$

Actually this field is independent on the chiral gauge and the scale parameter: we use again $\bar{M}=\widehat{\tilde{M}}$ and $\bar{M} M=M \bar{M}=\operatorname{det}(M)=r \mathrm{e}^{\mathrm{i} \theta}$. We let:

$$
\underline{M}=r^{-1 / 2} \mathrm{e}^{-i \theta / 2} M .
$$

We then get for any $M=s+\boldsymbol{u}+i \boldsymbol{v}+i p$ :

$$
\begin{aligned}
& M=\sqrt{r} \mathrm{e}^{i \theta / 2} \underline{M} ; \quad \bar{M}=\sqrt{r} \mathrm{e}^{i \theta / 2} \underline{M}=s-\boldsymbol{u}-i \boldsymbol{v}+i p \\
& r \mathrm{e}^{i \theta}=M \bar{M}=\sqrt{r} \mathrm{e}^{i \theta / 2} \underline{M} \sqrt{r} \mathrm{e}^{i \theta / 2} \underline{M}=r \mathrm{e}^{i \theta} \underline{M} \underline{\bar{M}} ; \quad \bar{M}=\underline{M}^{-1} ; \quad \underline{M} \in S L(2, \mathbb{C}), \\
& \mathcal{F}^{\prime}=M \mathcal{F} M^{-1}=\sqrt{r} \mathrm{e}^{i \theta / 2} \underline{M} \mathcal{F} r^{-1 / 2} \mathrm{e}^{-i \theta / 2} \underline{M}^{-1}=\underline{M} \mathcal{F} \underline{M}^{-1}=\underline{M} \mathcal{F} \underline{\bar{M}} .
\end{aligned}
$$

Like all gauge fields the quantized electromagnetic field $\mathcal{F}$ is insensitive to the chiral angle $\theta$ and the scale factor $r$. The $F_{(1)}$ and $F_{(2)}$ fields have value in the bivector part of $\mathrm{Cl}_{3}$ from (3.7) and (3.15). Then $\overline{F_{(1)}}=-F_{(1)}, \overline{F_{(2)}}=-F_{(2)}$ and we get:

$$
\bar{F}_{12}=\frac{1}{2}\left(\overline{F_{(1)} F_{(2)}+F_{(2)} F_{(1)}}\right)=\frac{1}{2}\left[\left(-F_{(2)}\right)\left(-F_{(1)}\right)+\left(-F_{(1)}\right)\left(-F_{(2)}\right)\right]=F_{12}
$$

Then this field is a scalar + pseudo-scalar field, similar to the Higgs field. More generally the field of an odd number of photons is a bivector field and the field of an even number of photons is scalar + pseudo-scalar field. This also implies that the quantized electromagnetic field is not reduced to the field $F=D^{-}(A)$ of the Section 3. The quantum field theory then identifies several fields which live in different linear spaces. The calculations of Clifford algebras allows this by using an addition of different scalar, vector and so on parts. And this is possible on the relativistic point of view because $\mathcal{F}^{\prime}=M \mathcal{F} M^{-1}$ is true in all cases. A third electromagnetic field is used in quantum physics, the "exterior" field $F$ created by the electric currents:

$$
\nabla \hat{F}=\frac{4 \pi}{c} j ; j=q J=\rho_{e}+j,
$$

where $\rho_{e}$ is the density of electric charge, $\boldsymbol{j}$ is the density of electric current an $J / \hbar c$ is the probability current created by the charged particle. Then (4.1) implies that the integration on the whole space of this density of charge is the charge of the particle, and the Pauli principle implies the orthogonality for two electrons. Then the normalization of each wave gives a total charge $2 e$, and the macroscopic conservation of the electric current results from the wave equations of quantum physics. 


\section{Concluding Remarks}

The unification of all interactions needs only two Clifford algebras, the $\mathrm{Cl}_{3}$ algebra of space which contains both a linear space isomorphic to the tangent space-time to the space-time manifold and the group of invariance generalizing the relativistic invariance. The greater $C l_{1,5}$ algebra introduces the eight $\phi^{1}, \cdots, \phi^{8}$. They are enough to get the $U(1) \times S U(2) \times S U(3)$ gauge group of the SM. In a further paper we shall explain how these algebras may be seen only from the intrinsic point of view of observers, us, who are living inside a sheet of space-time manifold.

We have resolved the wave equations of the electron + neutrino in the case of the hydrogen atom [15]. When the Dirac equation was solved and gave all the quantum numbers required, the true number of states and the true energy levels, this wave equation was definitely considered as the true relativistic wave equation of the electron. Now we got a new set of solutions for the three chiral spinors of the wave electron + neutrino, with exactly the quantum numbers required, the true number of states and the true energy levels. Nevertheless these solutions are not the solutions obtained with the wave equation of the alone electron. It is then possible to get the true experimental results from a deficient model. A necessary condition to get the awaited solutions seems the form $1 / r$ for the potential terms. This condition is satisfied for the exterior potential $A$ such as $F=\nabla \wedge \hat{A}$ where $F$ is the exterior field of (5.11).

A priori we do not expect a revolution with new particles or a new kind of interaction. The only new wave awaiting study is the magnetic monopole which uses the sixteenth $R^{8}$ spinor previously not used by the SM. If our interpretation of the three generations from the three $i \sigma_{k}$ terms present in our wave equations is true, we may expect three such magnetic monopoles with the respective proper mass of the electron, the muon and the tau. The magnetic monopoles of the second and the third generation should be able to disintegrate into magnetic monopoles of the first generation and it is possible that this is the reason of the activity during several hours after the electric discharges operated by L. Urutskoev and in the French laboratories [32]-[38].

Since only the fermion wave equations are doubly linked to their Lagrangian density, since the gauge fields do not have the same double link to the Lagrangian density and since the momentum-energy tensor is the conservative tensor linked to the invariance of this Lagrangian density under the space-time translations we can predict this: All momentum-energy belongs to the fermion part of the quantum wave, there is none momentum-energy belonging to the boson part of the quantum world. We do not see the momentum-energy of an isolated photon, we see only the momentum-energy emitted or absorbed by fermions. The double equality $E=m c^{2}=h v$ comes from two reasons: the first one was thought of by A. Einstein as a consequence of the fact that all energies have the same electromagnetic origin. The second equality comes from the invariance under a greater group than the Lorentz group (see [16] 4.1.2).

About anti-matter, we predict that any object made of anti-matter has the same gravitation as the corresponding object of matter. This comes from the wave equations for anti-particles which change only the sign of the differential term (see [16] 3.4). The 
momentum-energy tensor does not change and $T_{0}^{0}$ remains everywhere positive. Then, in conformity with all experiments, the total energy and the proper mass of any antiparticle are positive.

This work is not an end, it is a beginning. Now that we know the wave equations for all the first generation, the study of the two other generations must be done. It will be also necessary to resolve the equations and to confront the results with what has been learned in the laboratories. A great progress should be the description of the transition of an electron from one state to another one by emitting or absorbing a photon, allowing us to understand from where comes the probability of spontaneous and stimulated emission, the probability of absorption and the link with the number of photons in the vicinity of the electron.

What can we expect as experimental confirmation of this work? First the existence of leptonic magnetic monopoles is necessary in the $\mathrm{Cl}_{3}$ framework. The existence of such monopoles is already proved [33]-[38]. Next only one proper mass is available for the two quarks $d$ and $u$, and we can hope that our wave equations with mass terms and gauge terms depending on the different parts of the wave shall be able to account for the confinement of quarks in the protons and neutrons. Another exciting perspective is the fact that one complete wave is able to include the electron, the three quarks of a proton and the three quarks of a neutron, then to include a complete deuterium atom. Therefore an atom of helium, with two electrons, two protons and two neutrons, needs only two complete fermionic waves. This must be visible in nuclear physics, particularly when a photon is emitted by a whole atom. The numerous and precise results of both GR and SM may also be understood as a confirmation of this work, even if they have been obtained before and without us.

\section{References}

[1] Daviau, C., Bertrand, J. and Girardot, D. (2016) Journal of Modern Physics, 7, 1568-1590. https://doi.org/10.4236/jmp.2016.712143

[2] Naïmark, M.A. (1962) Les représentations linéaires du groupe de Lorentz. Dunod, Paris.

[3] Daviau, C. (1998) Annales de la Fondation Louis de Broglie, 23, 27-37.

[4] Daviau, C. (2005) Annales de la Fondation Louis de Broglie, 30, 409-428.

[5] Daviau, C. (2011) L'espace-temps double. JePublie, Pouillé-les-coteaux.

[6] Daviau, C. (2012) Advances in Applied Clifford Algebras, 22, 611-623.

[7] Daviau, C. (2012) Double Space-Time and More. JePublie, Pouillé-les-coteaux.

[8] Daviau, C. (2012) Nonlinear Dirac Equation, Magnetic Monopoles and Double SpaceTime. CISP, Cambridge.

[9] Daviau, C. (2013) Advances in Imaging and Electron Physics, 179, 1-136. https://doi.org/10.1016/B978-0-12-407700-3.00001-6

[10] Daviau, C. and Bertrand, J. (2014) Journal of Modern Physics, 5, 1001-1022. https://doi.org/10.4236/jmp.2014.511102

[11] Daviau, C. and Bertrand, J. (2014) Journal of Modern Physics, 5, 2149-2173. https://doi.org/10.4236/jmp.2014.518210

[12] Daviau, C. and Bertrand, J. (2014) New Insights in the Standard Model of Quantum Physics 
in Clifford Algebra. Je Publie, Pouillé-les-coteaux. http://hal.archives-ouvertes.fr/hal-00907848

[13] Daviau, C. (2015) Advances in Applied Clifford Algebras, 1-12.

[14] Daviau, C. and Bertrand, J. (2015) Journal of Applied Mathematics and Physics, 3, 46-61. https://doi.org/10.4236/jamp.2015.31007

[15] Daviau, C. and Bertrand, J. (2015) Journal of Modern Physics, 6, 1647-1656. https://doi.org/10.4236/jmp.2015.611166

[16] Daviau, C. and Bertrand, J. (2015c) The Standard Model of Quantum Physics in Clifford Algebra. World Science Publishing, Singapore.

[17] Lochak, G. (1983) Annales de la Fondation Louis de Broglie, 8, 345.

[18] Lochak, G. (1983) Annales de la Fondation Louis de Broglie, 9, 5.

[19] Lochak, G. (1985) International Journal of Theoretical Physics, 24, 1019-1050. https://doi.org/10.1007/BF00670815

[20] Lochak, G. (2004) Annales de la Fondation Louis de Broglie, 29, 297-316.

[21] Lochak, G. (2008) Annales de la Fondation Louis de Broglie, 33, 107-127.

[22] Lochak, G. (2010) Annales de la Fondation Louis de Broglie, 35, 1-18.

[23] Daviau, C. and Bertrand, J. (2015) Journal of Modern Physics, 6, 2080-2092. https://doi.org/10.4236/jmp.2015.614215

[24] Hestenes, D. (1973) Journal of Mathematical Physics, 14, 893-905. https://doi.org/10.1063/1.1666413

[25] Hestenes, D. (1986) A Unified Language for Mathematics and Physics \& Clifford Algebra and the Interpretation of Quantum Mechanics. In: Chisholm, J. and Common, A., Eds., Clifford Algebras and Their Applications in Mathematics and Physics, Reidel, Dordrecht, $1-23$.

[26] Boudet, R. (1995) The Takabayasi Moving Frame, from a Potential to the Z Boson. In: Jeffers, S. and Vigier, J., Eds., The Present Status of the Quantum Theory of the Light, Kluwer, Dordrecht.

[27] Boudet, R. (2011) Quantum Mechanics in the Geometry of Space-Time. Springer, Berlin.

[28] Fock, V. (1964) The Theory of Space, Time and Gravitation. Pergamum Press, London.

[29] Pauli, W. (1925) Zeitschrift für Physik, 31, 765-783. https://doi.org/10.1007/BF02980631

[30] Rose, M.E. (1960) Relativistic Electron Theory. John Wiley and Sons, Hoboken.

[31] Daviau, C. (1997) Advances in Applied Clifford Algebras, 7, 175-194.

[32] Daviau, C., Fargue, D., Priem, D. and Racineux, G. (2013) Annales de la Fondation Louis de Broglie, 38, 139-154.

[33] Urutskoev, L.I., Liksonov, V.I. and Tsinoev, V.G. (2000) Applied Physics, 4, 83-100.

[34] Urutskoev, L.I. and Liksonov, V.I. (2002) Annales de la Fondation Louis de Broglie, 27, 701-726.

[35] Urutskoev, L.I. (2004) Annales de la Fondation Louis de Broglie, 29, 1149-1164.

[36] Ivoilov, N.G. and Urutskoev, L.I. (2004) Annales de la Fondation Louis de Broglie, 29, 1177-1186.

[37] Priem, D., Racineux, G., Lochak, G., Daviau, C., Fargue, D., Karatchentzeff, M. and Lehn, H. (2008) Annales de la Fondation Louis de Broglie, 33, 129-138.

[38] Priem, D., Daviau, C. and Racineux, G. (2009) Annales de la Fondation Louis de Broglie, 34, 103-110. 
Submit or recommend next manuscript to SCIRP and we will provide best service for you:

Accepting pre-submission inquiries through Email, Facebook, LinkedIn, Twitter, etc. A wide selection of journals (inclusive of 9 subjects, more than 200 journals)

Providing 24-hour high-quality service

User-friendly online submission system

Fair and swift peer-review system

Efficient typesetting and proofreading procedure

Display of the result of downloads and visits, as well as the number of cited articles

Maximum dissemination of your research work

Submit your manuscript at: http://papersubmission.scirp.org/

Or contact jmp@scirp.org 